Meta

Journal des traducteurs

Translators' Journal

\title{
Aspects de la traduction audiovisuelle en Israël
}

\section{Francine Kaufmann}

Volume 43, numéro 1, mars 1998

La traduction et l'interprétation en Israël

Translation and Interpreting in Israel

URI : https://id.erudit.org/iderudit/003293ar

DOI : https://doi.org/10.7202/003293ar

Aller au sommaire du numéro

Éditeur(s)

Les Presses de l'Université de Montréal

ISSN

0026-0452 (imprimé)

1492-1421 (numérique)

Découvrir la revue

Citer cet article

Kaufmann, F. (1998). Aspects de la traduction audiovisuelle en Israël. Meta, 43(1), 130-141. https://doi.org/10.7202/003293ar

\section{Résumé de l'article}

Cet article brosse un tableau historique et socioculturel des médias israéliens et du contexte sociolinguistique dans lequel ils évoluent : société multilingue avec deux langues officielles (l'hébreu et l'arabe), pays d'immigration dont moins de la moitié des habitants possèdent l'hébreu comme langue maternelle, société neuve en expansion où l'État a longtemps gardé le monopole de la radiodiffusion mais où les médias audiovisuels connaissent une véri- table explosion depuis 1993. L'article passe en revue les procédés de transfert linguistique utilisés à la radio et à la télévision et en aborde quelques aspects : la prédominance du soustitrage, la formation des traducteurs, les normes de qualité linguistique. Il retrace les diverses étapes du sous-titrage à la télévision israélienne (l'ORTI).
Ce document est protégé par la loi sur le droit d'auteur. L'utilisation des services d'Érudit (y compris la reproduction) est assujettie à sa politique d'utilisation que vous pouvez consulter en ligne.

https://apropos.erudit.org/fr/usagers/politique-dutilisation/ 


\title{
ASPECTS DE LA TRADUCTION AUDIOVISUELLE EN ISRAËL
}

FRANCINE KAUFMANN

Université Bar-Ilan, Ramat-Gan, Israël

\begin{abstract}
Résumé
Cet article brosse un tableau historique et socioculturel des médias israéliens et du contexte sociolinguistique dans lequel ils évoluent: société multilingue avec deux langues officielles (l'hébreu et l'arabe), pays d'immigration dont moins de la moitié des habitants possèdent l'hébreu comme langue maternelle, société neuve en expansion où l'État a longtemps gardé le monopole de la radiodiffusion mais où les médias audiovisuels connaissent une véritable explosion depuis 1993. L'article passe en revue les procédés de transfert linguistique utilisés à la radio et à la télévision et en aborde quelques aspects: la prédominance du soustitrage, la formation des traducteurs, les normes de qualité linguistique. Il retrace les diverses étapes du sous-titrage à la télévision israélienne (l'ORTI).
\end{abstract}

\section{Abstract}

This article gives a historical and sociocultural perspective of the media in Israel--a multilingual society with two official languages (Hebrew and Arabic), a country of immigrants half of whom speak Hebrew as their first language, a young and rapidly growing society where broadcasting has long been state-controlled--which, since 1993 has witnessed a veritable audiovisual revolution. It reviews the linguistic transfer techniques and processes used in radio and television: subtitling, translator training, etc. It also looks at the various stages of subtitling in Israeli television.

\section{INTRODUCTION}

Dans l'État d'Israël, plusieurs facteurs jouent contre une politique linguistique uniforme dans le domaine de l'audiovisuel. Israël possède deux langues officielles : l'hébreu (langue maternelle de deux millions et demi d'habitants, mais parlé par près de quatre millions) et l'arabe (parlé par $20 \%$ de la population). Or l'hébreu, la langue nationale majoritaire, est une langue de faible diffusion. Les chaînes de télévision doivent donc traduire les produits audiovisuels en hébreu susceptibles d'être exportés ou présentés dans des festivals. Et comme dans la plupart des petits pays, la production audiovisuelle locale ne représente qu'une faible proportion de l'ensemble de la production "consommée", Israël est donc tributaire d'une forte proportion de programmes achetés à l'étranger qu'il faut traduire. Par ailleurs, Israël est un pays polyglotte (de par l'origine de sa population). Or en raison des taux particulièrement élevés d'immigrants (près de $40 \%$ ), beaucoup d'Israéliens sont incapables de suivre les sous-titres en hébreu à la télévision. Ils préfèrent donc souvent (comme beaucoup d'Arabes israéliens) chercher des dérivatifs sur les chaînes câblées en langues étrangères. Enfin, c'est au XIX ${ }^{\mathrm{e}}$ siècle que l'hébreu est redevenu une langue vivante, et le processus de son adaptation à la vie quotidienne et à la modernité n'est pas achevé : certaines lacunes sont particulièrement flagrantes en ce qui concerne la langue parlée (domaine de l'audiovisuel). Autant d'aspects qui offrent un vaste champ d'investigation et un défi pour les chercheurs.

Meta, XLIII, 1, 1998 
Pourtant, la traduction pour les médias audiovisuels ne constitue pas encore un domaine à part entière dans la recherche universitaire, en Israël. Certes, des articles importants ont été écrits, depuis une quinzaine d'années, par le professeur Raphaël Nir, du Département de communication et de presse de l'Université hébraïque de Jérusalem (Nir, 1983, 1984a et 1984b, 1994). Certes, une partie de ses disciples a réfléchi au sous-titrage (par exemple Brenner, 1983) ou à la place de la traduction en général dans la communication dite de masse (essentiellement pour la presse écrite). Certes, des cours ponctuels et des exercices de sous-titrage figurent depuis 1974 dans le cursus des étudiants de la section française de l'École d'interprètes et traducteurs de l'Université Bar-Ilan, et chaque promotion a pu visiter les services de sous-titrage de la télévision israélienne, le service d'information de la section française de Kol Israël (la radio nationale) ou le Jerusalem Post (quotidien israélien de langue anglaise et hebdomadaire de langue française). La visite s'accompagne d'une rencontre avec les responsables qui exposent aux étudiants les contraintes et les spécificités de la traduction pour les médias. Mais il ne s'est jamais agi de cours suivis et la maîtrise de traduction (option traduction audiovisuelle) reste encore un projet (qui deviendra peut-être réalité à la rentrée 1998). Il existe par contre, depuis 1990, un "atelier de traduction pour les médias électroniques" qui constitue un cours semestriel de formation théorique et pratique au sous-titrage de diverses catégories d'émissions, dans le cadre du Département de communication (Université de Jérusalem). Les deux enseignantes sont des sous-titreuses chevronnées de la télévision israélienne (Avigaill Neubach et Aya Gazit). Les étudiants travaillent sur un script après avoir visionné, en salle de vidéo, le film à traduire. Mais il s'agit d'un cours d'initiation (aujourd'hui, une heure et demie hebdomadaire au second semestre, essentiellement au niveau du premier cycle) qui n'a pas encore suscité de vocation universitaire.

En l'absence d'une bibliographie rigoureuse sur la traduction audiovisuelle en Israël, nous nous sommes résolue à offrir un état des lieux, une description issue d'une observation appuyée sur notre pratique professionnelle et sur un certain nombre d'études préliminaires (Kaufmann, 1979, 1993a et 1993b, 1995a et 1995b). Nous espérons ainsi offrir des éléments de comparaison avec d'autres pratiques nationales qui pourraient servir ultérieurement de matériel d'étude. La dernière partie sera consacrée à un entretien avec la responsable du sous-titrage à la télévision publique israélienne : Daphna Amit.

\section{LE PAYSAGE AUDIOVISUEL ISRAÉLIEN}

\subsection{La radio}

Pendant longtemps, le paysage audiovisuel israélien fut étonnamment rétréci. La colonie juive de Palestine (le Yichouv) s'était dotée, en 1936, d'un Service de radiodiffusion de la Palestine reconnu par l'autorité mandataire britannique : La voix de Jérusalem (Kol Yirouchalayim). Avec la création de l'État, en 1948, la station prit le nom de Kol Israël ("La voix d'Israël), diffusant en hébreu mais possédant une chaîne en arabe. En 1950, grâce à des fonds de l'Agence juive pour Israël, le réseau fut complété par un service d'émissions en langues étrangères (anglais, français, russe, mais aussi judéo-arabe maghrébin, yiddish, ladino, ou encore hongrois, roumain, persan, "hébreu facile", etc.) : Kol Tsion lagola ("La voix de Sion pour la Diaspora"). Ce service existe toujours et diffuse, sur ondes courtes, des émissions à destination (notamment) des principales communautés juives de l'étranger, mais aussi des émissions locales destinées aux nouveaux immigrants et aux touristes (bien que, depuis 1991, ces émissions locales soient diffusées désormais sur une chaîne spécialement réservée aux nouveaux immigrants : REKA (sigle de Réchète Kolétète Aliah : "la chaîne qui intègre les immigrants", consacrée en priorité aux émissions en russe et en amharique). Tous les journalistes et reporters des diverses 
sections se doivent d'être au moins bilingues (sans compter l'anglais, indispensable, en plus de l'hébreu). Ils traduisent et réécrivent eux-mêmes les dépêches d'agences et, pour les interviews, traduisent les passages retenus au montage et les enregistrent en voice-over. En cas de difficulté, ils s'adressent généralement à un collègue de la section voisine. Il en va de même pour les journalistes de Kol Israël en hébreu et en arabe ou de Galey Tsahal (la radio de l'armée), qui, jusqu'à ce jour, n'ont jamais fait appel à des traducteurs et qui ne recourent que très exceptionnellement à des interprètes, même pour les retransmissions en direct d'événements historiques comportant de longs discours ${ }^{1}$. En règle générale, les journalistes-vedettes préfèrent traduire eux-mêmes en direct, en les résumant, les points clefs des interventions des hommes politiques. Mais il y a tout de même des exceptions, et ce sont bien des interprètes professionnels auxquels on a fait appel pour interpréter (tant à la radio qu'à la télévision) les discours qui, par exemple, ponctuèrent la visite à Jérusalem du président Sadate (1977), la Conférence de paix de Madrid (1991) ou la première visite en Israël du président Clinton et son discours à la Knesseth (1995). Depuis 1995, la libéralisation des ondes a permis le développement de radios locales ou thématiques. Mais cette ouverture n'a pas changé, semble-t-il, les pratiques traductionnelles à la radio.

\subsection{La télévision}

\subsubsection{Historique}

La naissance de la télévision israélienne a eu lieu très tard, vingt ans après la création de l'État, en mai 1968. Il est vrai que David Ben-Gourion, Premier ministre fondateur de l'État, se méfiait du petit écran qu'il considérait comme un instrument d'abêtissement et un produit de luxe, trop dispendieux pour un petit État en construction. En outre, dans un pays d'immigration, accueillant des ressortissants de 102 nations différentes, où il est nécessaire d'éviter les frictions et les disparités, on redoutait les effets subversifs d'un médium encore mal dominé. La radio restait donc reine.

Pourtant, dès 1966, le ministère de l'Éducation et de la Culture, grâce à une donation de la fondation Rothschild, avait créé, à Ramat-Aviv, une télévision éducative (la Télévizia limoudite) diffusant des émissions scolaires et universitaires et diverses émissions culturelles, dont un tiers de production locale, d'excellent niveau. Le reste, essentiellement d'origine anglo-saxonne, était sous-titré, sauf les émissions d'animation, généralement doublés. Or en 1967, 100000 foyers possédaient déjà un poste de télévision qui, outre la Télévizia limoudite, captait essentiellement les émissions des pays arabes voisins (dont les programmes en hébreu (!), en anglais et en français de la seconde chaîne jordanienne). Et c'est notamment pour lutter contre la propagande arabe antisioniste (surtout après le rattachement soudain d'un million d'Arabes à Israël en 1967, après la guerre des Six Jours) que le gouvernement se décida à créer une télévision publique en hébreu et en arabe. Mais il continua à freiner considérablement l'introduction de la couleur (qui n'apparaît officiellement qu'en 1983) et il défendit jalousement son monopole télévisuel. La radio et la télévision furent intégrées dans le même service public: Rechout Hachidour, L'Office de radiodiffusion et télévision israélien, (l'ORTI; IBA en anglais: Israeli Broadcasting Authority).

Il faudra quinze ans de luttes (et près de sept ans de service "expérimental") pour voir naître, en 1993, une seconde chaîne indépendante, commerciale celle-là. Or, en 1993, pour échapper au monopole d'État, $20 \%$ des foyers israéliens étaient déjà câblés. Ils étaient $50 \%$ en 1997, tandis que parallèlement, près de 200000 antennes paraboliques équipaient les toits, capables de capter entre 60 et 150 chaînes différentes, le numérique n'étant plus très loin. 


\subsubsection{Une nouvelle réalité socioculturelle}

Avant d'aborder l'aspect pratique du transfert linguistique à la télévision, il faut d'abord rappeler que la traduction audiovisuelle en hébreu moderne, (langue "rare" ou "de faible diffusion" utilisée exclusivement en Israël) est peu rentable puisqu'elle s'adresse à un marché très limité; sur une population qui comptait, au seuil de 1995, 5,4 millions d'habitants, on dénombrait 4430000 Juifs, soit $81 \%$ de la population dont près de quatre millions parlent et écrivent la langue nationale, l'hébreu, mais 2,5 millions seulement en tant que langue maternelle. La seconde langue officielle, l'arabe, est parlée par près de $20 \%$ de la population et la première chaîne de télévision, tout en sous-titrant en arabe une grande partie des émissions en hébreu, consacre une plage horaire quotidienne aux émissions en arabe, ce qui représente une vingtaine d'heures hebdomadaires (souvent soustitrées à leur tour en hébreu). Au cinéma, la plupart des films sont sous-titrés, sur deux lignes, en deux langues.

Il est trop tôt pour apprécier l'impact de la mondialisation, de la déréglementation et des changements techniques intervenus depuis la fin du monopole d'État et de la chaîne unique en Israël (il y a moins de cinq ans), sans parler du développement de la télévision communautaire, du multimédia et du marché de la vidéo domestique. Notons encore que le processus de paix a favorisé les contacts internationaux d'Israël et son ouverture à d'autres influences (européenne, arabophone), remettant peut-être en cause la suprématie anglo-saxonne (et donc l'origine des émissions achetées).

Mais à n'en pas douter, l'explosion médiatique est en passe de modifier radicalement le paysage audiovisuel en Israël. Elle s'accompagne, depuis les dernières élections en Israël (29 mai 1996), d'une fragmentation visible de la société israélienne en micro-sociétés (Occidentaux/Orientaux, religieux/non pratiquants ou athées; nouveaux immigrants/ Israéliens de vieille souche; majorité juive/minorités arabe, druze, bédouine, chrétienne). Car, au lieu de poursuivre l'idéal du "creuset" (du melting-pot), la société "post-sioniste" se caractérise par une revendication identitaire qui aboutit à l'émergence de nouveaux partis politiques, dits "ethniques". Parallèlement, les minorités israéliennes ont tendance à s'équiper d'antennes paraboliques pour capter les chaînes qui parlent leur "langage" (linguistique et culturel : arabe, russe, éthiopien, mais aussi français, anglais, polonais, roumain, ou encore religieux, oriental, etc.). C'est pourquoi, le 9 mai 1997, le gouvernement israélien a décidé l'ouverture prochaine de cinq chaînes thématiques : une chaîne câblée en arabe, une chaîne pour nouveaux immigrants (en russe et en amharique), une chaîne sur la tradition juive, une chaîne d'informations et une chaîne de musique israélo-méditerranéenne, etc. Toutes les chaînes devront s'autofinancer par la publicité 2 .

Nul doute que dans un contexte de médias "thématiques" (et non plus nationaux), il faudra repenser prochainement la politique linguistique et traductionnelle d'Israël pour l'audiovisuel, surtout si l'on prend également en compte les changements sociolinguistiques qui marquent l'Israël de cette fin de siècle : d'une part l'augmentation de la proportion des Israéliens dont l'hébreu est la langue maternelle et principale -- et qui ne comprennent pas d'autres langues. (Ils ne représentaient que la moitié des Israéliens, en 1993.) C'est pour eux, par exemple que, depuis le $1^{\text {er }}$ janvier 1998, le magazine d'actualité international de CNN est diffusé tous les soirs à 21 heures, sur une chaîne câblée locale (la "neuf") avec des sous-titres en hébreu ${ }^{33}$. D'autre part, avec l'immigration massive des Juifs de Russie (10\% de la population actuelle), d'Éthiopie, mais aussi d'Amérique latine, d'Afrique du Sud, de France et d'ailleurs, environ $38 \%$ des juifs israéliens sont des nouveaux immigrants. Or si, à l'époque pionnière, les nouveaux venus pouvaient plus ou moins suivre la radio (ou écouter les émissions en langue étrangère), les immigrants d'aujourd'hui, à l'ère de la télévision, ont du mal à lire les sous-titres puisque l'hébreu, langue consonantique, ne note pas les voyelles de sorte qu'il faut une bonne maîtrise de la langue pour "reconstituer" 
les mots non vocalisés. D'où, pour éviter la "fuite" des télespectateurs, la diffusion, par les principales chaînes israéliennes, d'émissions en anglais, en russe ou avec un sous-titrage en russe, en amharique... Déjà, un magazine d'actualité de la télévision éducative (première chaîne) repasse en différé sur la seconde chaîne publique (éducative aussi) avec des sous-titres en russe.

Les sous-titres représentent également un handicap plutôt qu'une béquille pour les illettrés, les enfants, les malvoyants et les personnes âgées. (Pour les malentendants, il existe déjà une édition du bulletin d'information diffusée avec une interprétation en langue des signes, à 23 heures 30, sur la première chaîne.)

En tout cas, les besoins en traduction audiovisuelle, sous-titrage et peut-être, par la suite, doublage ou autres modalités -- telle la création de chaînes à canaux linguistiques distincts --, vont être décuplés. Actuellement, la plupart des films et des émissions étrangères sont sous-titrés sur deux lignes (en hébreu et en arabe, parfois en russe). Parallèlement, des chaînes thématiques juives commencent à acquérir les droits de diffusion des bulletins israéliens d'information du soir pour les diffuser à l'étranger (avec des sous-titres et des voice-over).

\section{LE SOUS-TITRAGE À LA TÉLÉVISION EN ISRAËL}

\subsection{La prédominance du sous-titrage}

Le choix du sous-titrage (et l'absence de doublage -- sauf pour les enfants) s'explique sans doute par des considérations à la fois économiques et culturelles. Aucune enquête sérieuse n'a été entreprise sur le sujet et il faut se contenter de témoignages subjectifs. Interrogé, un producteur (originaire d'Argentine) m'a expliqué que le coût d'une heure de sous-titrage se chiffrait, en 1995, à environ 300 dollars; le voice-over coûterait, à son avis, deux à trois fois plus cher, puisqu'il faut également louer un studio d'enregistrement et engager des comédiens (d'où, m'a-t-il dit, des problèmes syndicaux en perspective). Or "si pour l'Argentine (trente-trois millions d'habitants, dont la moitié sont analphabètes et ne peuvent pas lire des sous-titres), le doublage est rentable et performant, ce n'est pas le cas en Israël, petit pays avec une population de bientôt six millions d'habitants dont la moitié seulement sont hébréophones". Interrogé lui aussi, Na'hman Chaï, alors directeur de la seconde chaîne (commerciale), a constaté que le sous-titrage semble aujourd'hui, en Israël, le seul procédé naturel. Au point que l'interprétation simultanée dans un talk-show lui paraît impensable : à ses yeux, elle rompt le flux sonore et il reconnaît qu'il "déteste" !

Les contraintes objectives et les habitudes prises expliquent donc que la plupart des émissions "à traduire" sont sous-titrées. On assiste, ces dernières années, à une multiplication de sociétés qui se spécialisent dans le sous-titrage (et parfois le doublage), travaillant essentiellement pour le marché local, mais aussi pour l'exportation. Citons, parmi celles qui travaillent le plus souvent pour la télévision, Orion, Aley Kotéreth (à Jérusalem), Elrom (Kibboutz du Plateau du Golan, qui possède une antenne à Tel-Aviv) et une demidouzaine de sociétés à Tel-Aviv.

\subsection{Pour un contrôle de la qualité}

Ces sociétés recourent à des traducteurs indépendants (free-lance), dont la plupart reçoivent une formation sur le tas. Il n'existe pas, à notre connaissance, de formation maison systématique (sauf pour la télévision publique; cf. ci-dessous). Les exigences de qualité sont très variables, d'autant plus que les chaînes commerciales (qui n'ont pas de mission pédagogique) n'exigent pas un hébreu normatif. Les conditions de travail et les tarifs pratiqués n'encouragent pas toujours les traducteurs à investir les efforts nécessaires pour garantir une qualité acceptable, et bien qu'on ait tenté, il y a quelques années, de met- 
tre sur pied un syndicat des sous-titreurs, l'entreprise n'a pas eu de suite. Depuis 1997, Réchèt, l'une des sociétés produisant les émissions de la seconde chaîne, décerne un prix annuel de sous-titrage de films de cinéma et de télévision (le prix Yardéna Hare'el), ce qui aura sans doute un effet bénéfique sur les prestations obtenues ${ }^{4}$. Mais bien qu'on assiste à une amélioration constante de la production, les résultats laissent souvent rêveurs, et le public israélien s'amuse à relever les erreurs de traduction. Il existe même, dans la presse écrite, des rubriques qui signalent régulièrement les fautes les plus flagrantes ou les plus drôles (en général dans la traduction de l'anglais). C'est ainsi que l'hebdomadaire local Kol Haïr ("Toute la ville", de Jérusalem) publie chaque semaine une perle et une suggestion de traduction correcte sous le titre : Targoumon (nom d'un populaire dictionnaire électronique de poche). Réagissant aux lettres de lecteurs, le quotidien Yedioth A'haronoth consacrait de son côté, en 1995 et 1996, trois articles (sur une pleine page) aux "stupidités" relevées au petit écran. B. Mikhaël y concluait qu'il serait bon de rappeler aux sous-titreurs "qu'il ne suffit pas d'un dictionnaire anglais-hébreu, de doigts agiles et d'un clavier tolérant pour traduire un film. Encore faut-il une certaine dose de culture générale, de simple logique et de bon sens". Et il constatait qu'il existe une gradation dans la qualité du sous-titrage : "Sur la première chaîne [publique], la qualité des sous-titres est infiniment meilleure que sur les chaînes câblées et généralement meilleure que sur la seconde chaîne [commerciale ]". La chaîne familiale câblée (la 3) semble faire l'objet du plus grand nombre de critiques, sans doute parce qu'elle diffuse essentiellement des séries américaines (soap opéras, sitcoms, comédies, comme Seinfeld and Friends, Mad About You, Frazier...), qui exigent du traducteur une excellente connaissance de l'argot américain et de l'humour à la mode ${ }^{7}$. Mais les films diffusés sur l'ensemble des chaînes récoltent aussi des critiques.

\subsection{Le transfert linguistique à la télévision "publique"}

La situation est différente sur la première chaîne de télévision qui continue à obéir à des critères plus stricts, étant chargée par la loi, en tant que "service national", de remplir des missions éducatives et culturelles (de "développer la culture et répandre la connaissance", de "promouvoir les objectifs de l'Éducation nationale") tout en restant un instrument de "divertissement et d'information". Rien d'étonnant, donc, si le langage employé à la radio et à la télévision publiques est strictement réglementé par un conseiller linguistique reflétant les positions de l'Académie de la langue hébraïque ${ }^{8}$.

La première chaîne recourt presque exclusivement au sous-titrage (sauf, nous l'avons vu, dans les films d'animation pour enfants, qui sont diffusés par la télévision éducative et sont "doublés", ou lors de certains événements historiques retransmis en direct, avec interprétation simultanée ou avec des résumés intermittents de l'essentiel du message, généralement effectués par les journalistes et reporters plutôt que par des interprètes). L'interprétation simultanée n'est pratiquée par des interprètes professionnels que dans des cas rarissimes, essentiellement dans des talk-shows où sont réunis plusieurs invités et où l'on interprète ce qui se dit en studio pour le ou les invités qui ne comprennent pas l'hébreu. Mais la plupart des interviews seront réalisées en anglais (très rarement dans une autre langue) et traduites en consécutive par l'animateur lui-même (ou sous-titrées s'il s'agit d'une séquence préparée à l'avance ou d'une émission en différé). L'habitude qui prévaut dans certains pays de traduire oralement en voice-over (doublage synchrone) les interviews et les commentaires dits partiellement en voix hors champ (voix off) est pratiquement inexistante en Israël. Si l'on a longtemps accepté le voice-over pour les commentaires, l'on assiste à une régression et à son remplacement progressif par des sous-titres : sans doute pour des raisons de coûts et parce que, depuis l'introduction des logiciels de sous-titrage, ce dernier procédé est devenu plus simple et plus rapide. Peut-être aussi parce que l'œil du télespectateur israélien est habitué au sous-titre et que -- nous l'avons vu -- de 
plus en plus d'Israéliens possèdent l'hébreu comme langue maternelle et peuvent déchiffrer en un clin d'œil l'alphabet non vocalisé, tandis que l'oreille du télespectateur est habituée à écouter les émissions en version originale. (Rappelons que beaucoup d'Israéliens comprennent l'anglais ainsi que d'autres langues acquises dans leur pays d'origine ou dans leur milieu familial et professionnel.)

\subsection{Les quatre services de traduction à l'ORTI}

Il existe quatre services s'occupant de transfert linguistique à la télévision publique. Il y a d'abord le service des exportations, chargé de vendre des films et des émissions israéliennes à l'étranger ou de les présenter dans des festivals et des foires. Nous n'en parlerons pas ici sinon pour dire que l'essentiel des traductions et de la saisie est réalisé à l'extérieur de l'ORTI par des sociétés de sous-traitance (de type Manpower) ou par des pigistes. Il en va de même des films et des émissions diffusés par la section arabe. Cette dernière s'adresse à des sociétés externes spécialisées dans les traductions de et vers l'arabe (cf., dans ce même numéro, l'article d'Avraham Robinson). Il existe par contre deux services qui font réaliser le travail de sous-titrage sur place ou qui contrôlent étroitement l'ensemble des phases de la production. Il s'agit du service des informations, qui dispose d'une équipe de permanents et de pigistes contactés quotidiennement, vers midi, après la réunion du comité de rédaction du journal télévisé diffusé à vingt heures. C'est alors que les séquences d'actualités achetées à l'étranger et les interviews réalisées en Israël et dans les territoires en langues étrangères (essentiellement l'arabe et l'anglais) sont connues ou prévisibles. On convoque alors l'équipe du jour (en fonction du volume à traduire et des combinaisons linguistiques souhaitées). Il arrive aussi qu'il faille traduire des émissions qui parviennent à la télévision très peu de temps avant la diffusion prévue ${ }^{9}$ et ne peuvent être traduits à loisir par le quatrième service concerné -- de loin le plus important --, le service de traduction du Département des achats (Seratim kenouyim, littéralement "films achetés", en anglais : Acquired Films Department). Il nous reste donc à découvrir la manière dont se pratique le plus couramment le sous-titrage à la télévision israélienne, dans le cadre du Département des achats. La dernière partie de cette contribution sera constituée par un entretien sous forme de questions et réponses avec la responsable du sous-titrage pour la première chaîne, Daphna Amit (entretien réalisé en hébreu le 17 janvier 1996).

\section{ENTRETIEN AVEC DAPHNA AMIT ${ }^{10}$}

Francine Kaufmann: Comment définiriez-vous votre fonction et comment a-t-elle évolué?

Daphna Amit : Lorsque je suis entrée au Département des achats, en 1972, la télévision venait d'être créée quatre ans auparavant. L'on m'a proposé un poste à mi-temps de "responsable de la langue de traduction”. Je devais réviser les sous-titres, mais aussi répartir le travail à réaliser entre les traducteurs. Néanmoins, il n'a jamais existé de service de traduction. Notre département est chargé d'acheter les films, de les préparer en vue de la diffusion et de veiller au bon fonctionnement de la diffusion. Au cours des diverses phases de la préparation, s'inscrit la phase de traduction. Je suis employée aujourd'hui à plein temps, titularisée et chargée du choix des traducteurs, de leur formation, de la distribution des tâches et de la révision du matériel traduit. Nous avons environ 25 traducteurs, tous indépendants (vacataires). Mais le département compte aussi (en plus du directeur, de son adjoint et de trois employées de bureau) un responsable de la réception et de l'envoi du matériel acheté, un technicien chargé du transfert technique des films (en beta, quart de 
pouce ou demi-pouce) et plusieurs responsables de la diffusion des sous-titres : deux ou trois permanents et une dizaine d'indépendants.

F.K. : Quelles sont les différentes phases du sous-titrage et en quoi consiste le travail de ceux que vous appelez les "responsables de la diffusion" (mèviim lechidour)?

D.A. : Ils font partie du personnel de post-production. Ils reçoivent le matériel à traduire du directeur du département. Ils veillent à le faire transférer sur des cassettes de travail. Ils effectuent le découpage du texte en unités de sous-titrage d'environ trois à quatre secondes. Ils contrôlent l'adéquation du script (s'il accompagne le film) avec les dialogues et autres séquences verbales qui figurent dans le produit fini (et pour cela, il est indispensable qu'ils connaissent une ou plusieurs langues étrangères). Généralement, le script est assez fidèle. Si ce n'est pas le cas, ils engagent un pigiste chargé de "sortir" les dialogues à partir de la bande sonore. Je voudrais souligner que dans la plupart des sociétés commerciales, le traducteur est appelé à "sortir" lui-même les dialogues. Mais notre principe est de fournir au traducteur un texte aussi exact que possible tout en multipliant les "oreilles" intermédiaires, afin de garantir une meilleure qualité. Il arrive néanmoins que le traducteur ait meilleure "oreille" que ceux qui l'ont précédé, qu'il "reconnaisse" des mots ou des expressions mal identifiés et qu'il les corrige.

C'est à moi qu'il appartient de choisir le traducteur le plus à même de traduire le programme ou le film en fonction de divers paramètres (langue, catégorie de matériel, problèmes spécifiques). Le responsable de la diffusion organise alors une séance de visionnement (sur copie U-Matic, qui permet de travailler sur le film sans le rayer) et remet le script au traducteur. Celui-ci prend des notes personnelles pendant le visionnement afin de préciser certains détails nécessaires à la levée des ambiguïtés (prononciation des noms, genre du locuteur, situation). Mais le traducteur travaille chez lui, avec le script découpé et devant son ordinateur. En cas de doute, il peut demander un nouveau visionnement. Une fois la traduction effectuée, je la révise avant de la remettre au responsable de la diffusion chargé de la faire parvenir au service arabe. En effet, le sous-titre arabe doit se conformer au sous-titre hébraïque, puisque la majorité absolue des émissions sont soustitrées dans les deux langues, sur deux lignes superposées. L'ORTI est un organe officiel qui doit respecter les deux langues officielles de l'État, l'hébreu et l'arabe. En cela, nous différons des chaînes commerciales qui ne sous-titrent pratiquement qu'en hébreu et peuvent bénéficier de deux lignes. Chez nous, on ne renonce à l'arabe que dans des cas exceptionnels, lorsqu'un sous-titrage sur deux lignes porterait irrémédiablement atteinte à la qualité de la traduction, par exemple pour des pièces de Shakespeare ou des séries comiques fondées essentiellement sur des jeux de mots. Dès que les sous-titres dans les deux langues sont prêts, le responsable de la diffusion réalise l'incrustation synchrone du matériel sur le support qui servira à la diffusion. Lors de cette dernière phase, il s'assure une dernière fois que chaque sous-titre est bien conforme à ce qui se dit dans le film. Éventuellement, il corrige aussi l'orthographe et la typographie. Autrefois, lorsque les sous-titres n'étaient pas incrustés sur la copie-antenne (l'original étant renvoyé) mais qu'on diffusait l'original en projetant, en surimpression, les sous-titres (ce qui laissait l'original intouché), c'était le responsable de la diffusion qui introduisait manuellement chaque sous-titre en pressant un bouton pour faire apparaître le nouveau segment ${ }^{11}$. Aujourd'hui, la copie incrustée est prête à la diffusion. Mais le responsable de la diffusion reste tout de même en régie au moment de l'émission pour intervenir en cas d'incident technique et introduire manuellement le sous-titre sur une copie de sécurité, si l'ordinateur s'emballe. (Là encore, il faut qu'il comprenne la langue du matériel diffusé.)

F.K. : Quels sont les changements techniques qui ont le plus marqué votre travail, depuis que vous êtes entrée à la télévision? 
D.A. : Les bouleversements ont été considérables, et ce serait trop long de les énumérer. Mais le plus flagrant a été la mise au point, après des efforts patients et laborieux, d'un logiciel de sous-titrage qui répond aux exigences spécifiques de notre département. Aujourd'hui, nous en avons équipé tous nos traducteurs ainsi que notre bureau. Il nous épargne, bien sûr, la nécessité fastidieuse de compter les 25 ou 26 signes qui composent en moyenne chaque sous-titre, puisqu'il se bloque et fait entendre un bip dès que le quota de la ligne est atteint. Mais il permet aussi d'augmenter légèrement le nombre de signes, notamment en contractant les espaces. Il facilite les échanges entre moi (le réviseur) et les traducteurs. La communication est entièrement informatisée entre nous et la révision se fait sur ordinateur. Plus besoin de tirage papier. Plus besoin de retaper le texte avant la diffusion. Le traducteur remet une disquette. L'ordinateur réduit ainsi de moitié la durée du travail. Il a surtout l'avantage de servir à la révision du texte et à la formation des traducteurs. Il permet d'introduire une marque spécifique pour indiquer soit un changement, une correction, soit une remarque, une annotation. Je pourrai bientôt extraire toutes les remarques et les envoyer aux traducteurs. Par ailleurs, ce matériel peut servir à l'enseignement et à la recherche, car il conserve "l'histoire" du texte, devenant une "mémoire de traduction". Le logiciel permet en effet d'enregistrer les trois états antérieurs du texte (s'il y en a quatre, le plus ancien est éliminé). On peut ainsi apprécier les hésitations, les problèmes rencontrés, comparer les variantes, tirer sur imprimante tous les passages litigieux ou difficiles signalés par une marque spécifique.

F.K. : Comment s'effectuent le recrutement et la formation des traducteurs?

D.A. : En général, les traducteurs s'adressent à nous. J'inscris, sur des formulaires, les informations qui me semblent pertinentes concernant le postulant. Les diplômes universitaires ne sont pas forcément un critère déterminant. En revanche, le fait d'avoir vécu suffisamment longtemps dans un autre pays me paraît plus important. En effet, la langue des films et des séries télévisées est, dans la plupart des cas, une langue parlée, voire argotique, qu'on n'acquiert pas dans les universités mais par un contact direct et personnel. Environ une fois tous les deux ans (en fonction des besoins de notre département), je reprends tous les dossiers reçus (entre cent et cent cinquante demandes en deux ans) et je sélectionne 25 à 30 candidats, que je convoque à un examen. Cet examen est composé de deux parties : un contrôle des connaissances de la langue hébraïque grâce à une liste de cinquante phrases à corriger ou à améliorer parce qu'elles présentent des problèmes d'orthographe, de genre (féminin/masculin), de prépositions, des calques, des emprunts, un ordre des mots défectueux, etc. La seconde partie consiste à traduire des extraits de dialogues empruntés à des émissions ou films déjà diffusés représentant divers niveaux de langue : langue parlée, langue soutenue, etc. Je retiens cinq candidats en moyenne et je les réunis trois ou quatre fois pour des séances de formation en groupe. Ensuite, je travaille avec chacun individuellement.

F.K. : Quels sont les tarifs pratiqués par l'ORTI? Quel volume de travail est traité par votre département et quelles sont les langues privilégiées?

D.A. : Le tarif actuel (janvier 1996) est de 64 agoroth pour un sous-titre d'une ligne (l'écrasante majorité d'entre eux puisque la seconde ligne est réservée à l'arabe) et de 72 agoroth pour un sous-titre de deux lignes (soit environ un franc français ou vingt cents américains le sous-titre d'une ligne). La traduction des commentaires se paie à la minute (un peu plus de quatre dollars la minute). Quant au volume du travail, il est variable. Il a diminué ces derniers temps, car nous sommes entrés dans l'ère de l'audimat et de la concurrence. Aussi nous distinguons-nous par un effort pour promouvoir les créations originales (en hébreu). Et depuis que l'ORTI a créé une filiale sur le câble (la chaîne 33, plus populaire), le gros de la production étrangère achetée est diffusé sur la chaîne 33. Actuellement, notre départe- 
ment sous-titre environ 25 à 30 heures de programmes par semaine. La majeure partie du matériel est en anglais (américain ou britannique), puis viennent le français, l'espagnol et l'italien. Il arrive, bien sûr, que nous traitions des films tchèques, polonais, etc.

F.K. : Donnez-vous des directives très strictes à vos sous-titreurs quant au choix du niveau de langue? Je pense au fait que vous êtes une chaîne nationale et que vous devez respectez certaines normes. Qui fixe ces normes?

D.A. : L'ORTI est un service public et remplit une mission éducative en contribuant à la diffusion d'un hébreu correct, normatif. Durant bien des années, nous suivions les directives du conseiller linguistique de l'ORTI, feu Abba Ben-David, qui à sa manière avait créé des normes tendant au purisme ${ }^{12}$. Son successeur, Ruth Almagor-Ramon, adopte une attitude plus souple, plus conforme à l'esprit du temps. Mais chaque station possède son propre conseiller linguistique: Nathan Na'hmias pour la télévision éducative, Reouma Yits'haki pour la seconde chaîne, avec une conseillère spéciale pour le département de l'information : Margalith Guez, homologue de Dvora Ben-Israël, conseillère de la première chaîne pour les actualités. Les chaînes câblées (et les stations de radio) ont aussi leurs conseillers. Et il convient de signaler que tous les deux mois (en moyenne), se tient une réunion de l'ensemble des conseillers pour discuter des problèmes qui leur sont communs. C'est, à ma connaissance, le seul domaine où toutes les chaînes coopèrent et oublient leur concurrence sur le terrain, afin de mieux servir les intérêts de la diffusion de l'hébreu correct et des néologismes recommandés. Tous tentent d'harmoniser leurs positions. Bien sûr, c'est l'Académie de la langue hébraïque qui détermine les normes du bon usage, qui décide de ce qui est correct et de ce qui ne l'est pas. Les conseillers linguistiques (dont certains sont des employés de l'Académie) respectent les décrets, mais chacun les interprète à sa manière, avec une certaine dose de subjectivité ou en fonction des aspects spécifiques de sa chaîne. Dans notre département, nous essayons de respecter les recommandations de l'Académie dans la mesure du possible. Mais tout dépend du type de film à traduire. S'il se caractérise par une langue soutenue, nous employons une langue soutenue. Mais en général, les dialogues, dans un même film, appartiennent à des registres différents de la langue parlée. Compte tenu des contraintes techniques imposées par le sous-titrage, la traduction tente de refléter les divers niveaux de langue de l'original. Lorsque la langue de l'original reflète un parler incorrect, c'est moi qui juge des écarts permis vis-à-vis de l'hébreu normatif. Mais chaque traducteur est censé connaître le bon usage et les écarts courants. Je les forme aussi dans ce domaine.

F.K. : Pouvez-vous dire un mot des divers procédés de transfert linguistique employés en Israël et pouvez-vous expliquer la prééminence du sous-titrage?

D.A. : Israël recourt essentiellement au sous-titrage pour des raisons de coûts. Il en va de même dans la plupart des pays qui comptent une population de moins de huit millions d'habitants, comme la Hollande, la Grèce, le Danemark... Sauf erreur, le doublage coûte dix fois plus cher que le sous-titrage : il faut engager des acteurs ou des speakers, louer un studio d'enregistrement. On ne recourt donc au doublage que pour les programmes destinés aux enfants d'âge préscolaire, qui ne savent pas encore lire. Quant à l'interprétation simultanée, on l'utilise très rarement : elle est moins exacte que d'autres procédés. Elle est impensable pour des films, des séries ou des émissions et ne s'utilise éventuellement que pour des interviews dans des émissions d'actualité. Quant au voice-over, on l'utilisait autrefois dans les programmes étrangers présentés par un speaker. On y a renoncé, car c'est un procédé long et coûteux.

F.K. : Pour conclure, qu'est-ce qui, à vos yeux, est spécifiquement israélien dans votre travail de sous-titrage pour une chaîne nationale? 
D.A. : Je relèverai trois points. 1) La nécessité de préparer un sous-titrage bilingue (une ligne par langue) constitue une contrainte particulière, qui exige une condensation plus stricte encore que d'habitude. 2) La langue hébraïque est encore en pleine effervescence et ne cesse d'évoluer. Le processus de régénération n'est pas achevé et les débats se poursuivent sur les structures grammaticales, les tournures correctes... Il nous manque encore bien des mots et il faut se résoudre à recourir souvent à des emprunts aux langues étrangères. La langue parlée évolue très rapidement et subit l'influence, notamment, de l'argot militaire. Les traducteurs réagissent subjectivement à l'apparition de telle ou telle expression et ils n'ont pas la même attitude devant l'argot. Il est parfois difficile de déterminer l'équivalent le plus proche, en hébreu, d'un terme de la langue parlée. 3) Plus de la moitié de la population israélienne (la génération des parents et les nouveaux immigrants) ne réussit pas à suivre les sous-titres. L'hébreu n'est pas leur langue maternelle et ils ont du mal à déchiffrer cet alphabet qui reste pour eux difficile.

F.K. : Par contre, les jeunes apprennent les langues étrangères et améliorent leur connaissance de leur propre langue en lisant les sous-titres. Aviva Raz, l'une des sous-titreuses de votre section, m'a rapporté que lors d'un récent symposium organisé pour les traducteurs de l'ORTI, l'un des orateurs (un membre de l'Académie hébraïque) leur a déclaré : "On se plaint en général que la jeune génération ne lit plus. J'affirme que les jeunes lisent toujours autant : ils lisent les sous-titres à la télévision."

\section{Notes}

1. Ces données mériteraient d'être vérifiées scientifiquement. Elles s'appuient sur l'expérience de douze années de travail à Kol Israël (1973-1985).

2. Cf. le reportage de Yossi Avissar pour Mabat chèni, $1^{\text {re }}$ chaîne, juin 1997.

3. Cf. l'article de 'Haïm Bar'am dans Kol Haïr (Jérusalem) du 2 janvier 1998.

4. Cf. Targuima, $\mathrm{n}^{\circ}$ 37, novembre 1996, p. 8, et circulaire de décembre 1996. En 1997, le prix a porté sur le sous-titrage de l'anglais à l'hébreu de films de fiction pour la télévision et le cinéma, diffusés à la télévision (nationale, commerciale ou câblée) dans les trois années écoulées. Quatre sous-titreuses ont été distinguées : deux se sont partagé le premier prix (de 10000 sheqels); deux autres ont reçu une mention spéciale. La société Rèchèt (Herzlia) se propose de modifier chaque année les catégories concernées (cinéma ou télévision, genre dramatique, langue de départ, "âge" de l'œuvre diffusée).

5. B. Mikhaël, Yedioth A'haronoth du 9 février 1996, p. 22. Parmi les principales fautes relevées, il signale la confusion entre divers synonymes proposés par le dictionnaire. Il cite aussi les sous-titres de l'oratorio de Haendel Le Messie, sur la chaîne 8 (câble culturel). Dans son "ignorance crasse, le pauvre traducteur ignorait que toutes les phrases de l'oratorio étaient empruntées à l'Ancien et au Nouveau Testament. Si bien qu'il a été amené à "réécrire" la Bible !" (ibid.).

6. B. Mikhaël, Yedioth A'haronoth du 31 mai 1996, p. 25. L'auteur cite une lettre émanant d'une lectrice, ellemême sous-titreuse de la première chaîne (A.R., sans doute Aviva Raz). Mais il reconnaît qu'elle dit vrai.

7. Cf. la mise en garde du magazine de Haaretz (5 décembre 1997) avant la rediffusion quotidienne de la série Frazier sur la 3. Parmi les perles relevées : "I see a lot of hors-d'œuvre" est traduit en hébreu par :"Je vois beaucoup en dehors du travail"; "group therapy" est rendu par "un groupe de soins", etc. Cf. aussi, dans la rubrique télévision du Jerusalem Post (12 au 18 décembre 1997) ce cri du cœur de Calev Ben-David citant des bourdes vues dans les sitcoms diffusées sur la 3 : "I say it's time we stand up for our sabra kinsmen and demand a better Hebrew subtitles on English shows. And the next time an Israeli tells you he doesn't see what's so funny about Seinfeld, remember that, even more than poetry, it's humor that's lost in translation."

8. Articles 2 et 3 de la loi du 17 mars 1965 sur l'ORTI ('Hok rechout hachidour, plusieurs fois amendée depuis). Cf. Kaufmann 1979, pp. 65-66.

9. C'est le cas d'une mémorable interview en arabe du roi Hassan II du Maroc, réalisée par Ehoud Yeari, au moment de la Conférence de Casablanca. Elle devait être relayée en duplex par la télévision marocaine le 21 octobre 1994, dans le cadre du magazine d'actualité hebdomadaire de la première chaîne (Yoman, diffusé chaque vendredi de $20 \mathrm{~h}$ à $21 \mathrm{~h}$ ). Les télespectateurs marocains devaient suivre l'interview de leur roi sous-titrée en hébreu. Or les sous-titres ne furent pas prêts à temps. Mais cette interview exclusive avait été annoncée à grand renfort de publicité. On diffusa donc en duplex la partie de l'interview déjà sous-titrée au début du journal et l'on annonça aux télespectateurs que la suite de l'interview serait donnée en fin de magazine, dès que le reste des sous-titres seraient prêts. Ce qui fut fait. (Cf. la rubrique télévision d'Avraham Tal dans Haaretz du 23 octobre 1994.) 
10. Née en 1947 à Binyamina (Israël), Daphna Amit est diplômée de l'Université hébraïque de Jérusalem en langue et littérature hébraïques et en littérature anglaise. De 1971 à 1973, elle travaille dans l'équipe "langue moderne et contemporaine" du Dictionnaire historique de l'Académie de la langue hébraïque. Depuis 1972, elle travaille à la télévision israélienne où elle est responsable du sous-titrage (formation des traducteurs, répartition des tâches et révision des sous-titres). Depuis 1987, elle travaille parallèlement pour l'édition : elle a traduit une quinzaine de romans et essais et une trentaine d'articles (dont une quinzaine sur la "formation à la réflexion").

11. Les techniciens du Département du développement de l'ORTI avaient mis au point, en 1980, une "machine à sous-titres" composée de deux éléments, l'un réservé à la saisie des sous-titres hébreux et arabes enregistrés sur un petit disque mais visualisés sur un écran. Le responsable de la diffusion pouvait encore introduire des corrections de dernière minute. Le second élément servait à la diffusion par surimpression à l'antenne. Le disque étant engagé dans la machine, une simple pression sur un bouton introduisait le nouveau sous-titre ("prévisualisé" sur le second écran). Le relâchement de la pression le faisait disparaître. Le responsable de la diffusion pouvait varier le rythme, sauter plusieurs sous-titres en cas d'incident technique sans que le défilement apparaisse à l'écran. Cette machine avait grandement facilité le sous-titrage puisque, auparavant, les sous-titres étaient tapés sur un rouleau de papier qui se déroulait à la diffusion. Chaque incident, chaque erreur à corriger exigeait l'intervention d'un technicien. (Cf. Mira Kney-Paz dans Kol Haïr-Jérusalem, 19 février 1982.)

12. Cf. dans ce même numéro l'article de Moché Bar-Acher (section 2.3) sur les relations entre l'Académie de la langue et l'ORTI. Notons que le légendaire Abba Ben-David, qui fut durant plusieurs décennies le conseiller linguistique de l'ORTI, avait rédigé un manuel devenu la référence obligée de tout collaborateur de l'ORTI: Madrikh lechone haradio vehatélévizia (Guide de la langue de la radio et de la télévision). Abba Ben-David est mort à Jérusalem le 28 décembre 1984, à l'âge de 84 ans.

\section{RÉFÉRENCES}

BEN-DAVID, Abba (1974) : Madrikh lechone haradio vehatélévizia (Guide de la langue de la radio et de la télévision), Rechout Hachidour, merkaz hahadrakha (publication du Centre de formation de l'ORTI), Jérusalem, $370 \mathrm{p}$

BRENNER, Eylat (1983): Tirgoum sirtey televizia (La traduction de films pour la télévision), mémoire de maîtrise sous la direction du Pr. Raphaël Nir et de Chochana Blum-Kulka, Université de Jérusalem, 27 p.

GAZIT, Aya (à paraître) : "Mimeafyeney hatirgoum liktouviyoyh" (Quelques caractéristiques du sous-titrage), communication devant l'Institut Masie, Académie de la langue hébraïque, Jérusalem (16/12/96).

KAUFMANN, Francine (1979): "Israël à l'ère de la télévision (l'impact de la télévision sur la société israélienne)", in $Y O D, \mathrm{~V}$ (1), Paris, INALCO, Université Paris III, pp. 63-81.

KAUFMANN, Francine (1993a) : "Un rituel de la mémoire : Le "Jour de la Shoa" dans les médias israéliens, l'exemple de 1992", Pardès, 18, Paris, éd. du Cerf, pp. 48-65.

KAUFMANN, Francine (1993b): "Interprétation consécutive et interview dans le film Shoah de Claude Lanzmann", Meta, 38 (4), pp. 664-673.

KAUFMANN, Francine (1995a) : "Formation à la traduction et à l'interprétation pour les médias audiovisuels", Translatio, XIV (3-4), Actes du colloque de Strasbourg, Comité Médias de la FIT, EST, Conseil de l'Europe, Yves Gambier (dir.), pp. 431-443.

KAUFMANN, Francine (1995b) : "Place et perspectives de l'interprétation simultanée et consécutive dans les actualités radiophoniques", Translatio, XIV (3-4), Actes du colloque de Strasbourg, Comité Médias de la FIT, EST, Conseil de l'Europe, Yves Gambier (dir.), pp. 363-375.

NIR, Raphaël (1983) : "Darkey hatserikha chel hatirgoum haïvri bessirtey televizia meyoubaïm" (Les modes de consommation de la traduction en hébreu des films de télévision importés), Mélanges David Gross, Jérusalem, pp. 186-195.

NIR, Raphaël (1984a): "Linguistic and sociolinguistic problems in the translation of imported TV films in Israël”, The International Journal of Sociology of Language, 48, pp. 81-97.

NIR, Raphaël (1984b) : "Hatirgoum bessirtey televizia" (La traduction des films de télévision), Lachon, mediyoum oumesser : iyounim bassiguenon ouvaretorika chel hatikchoret hahamonit beYisrael (Language, Medium and Message : Studies in the Style and Rhetoric of the Israeli Mass Media), Jérusalem, Posner and Sons, pp. 135-170.

NIR, Raphaël (1994) : "Keriya, tsorekh, tsfiya - ktouviyoth : hatirgoum kessouga chel tekstim" (Lecture, besoin, spectacle -- sous-titres : la traduction comme genre de textes), Yad lakore, vol. XXVIII, n 1-2, pp. 3-7. 